\title{
TRASTORNOS DE LA PERSONALIDAD Y CONDUCTA INTERPERSONAL: UN ANÁLISIS DIMENSIONAL A PARTIR DEL CIRCUMPLEX INTERPERSONAL
}

\author{
ELENA FELIPE ${ }^{1}$ y ALEJANDRO Ávila ${ }^{2}$ \\ ${ }^{1}$ E.U. Enfermería y Terapia Ocupacional, Universidad de Extremadura, Cáceres \\ ${ }^{2}$ Facultad de Psicología, Universidad Complutense de Madrid
}

\begin{abstract}
Resumen: El Modelo circumplex de Las Escalas de Adjetivos Interpersonales (IAS, Wiggins, 1996) es un modelo integrador que permite la investigación de los contenidos interpersonales asociados a las categorías de trastornos de la personalidad, mediante la análisis sustantivo del contenido interpersonal de esas categorías psicopatológicas. En este estudio pretendemos explorar las relaciones entre el circumplex interpersonal de J.S. Wiggins y las escalas de trastornos de personalidad en sujetos clínicos y aprovechando las propiedades geométricas del círculo de variables. Utilizamos el Inventario Clínico Multiaxial de Millon (MCMI-II) para evaluar las escalas de trastornos de la personalidad y las IAS como marcadoras del espacio interpersonal. Encontramos que muchos de las escalas de trastornos de la personalidad medidas por el MCMI-II pueden ser identificadas en el circumplex IAS.
\end{abstract}

Palabras clave: Trastornos de la personalidad, conducta interpersonal, Escalas de Adjetivos Interpersonales (IAS), circumplex interpersonal.

\section{Personality disorders and Interpersonal behavior: a dimensional analysis based on the Interpersonal Circumplex}

\begin{abstract}
The circumplex model of Interpersonal Adjective Scales (IAS, Wiggins, 1996) is an integrative model which allows research into the interpersonal contents associated with the categories of personality disorders, through the substantive analysis of the interpersonal contents of these psychopathological categories. This study purports to explore the relationship between the Interpersonal Circumplex of J.S. Wiggins and the scales of personality disorders in clinical subjects and taking advantage of the geometrical properties of circle variables. We used the Millon Clinical Multiaxial Inventory (MCMI-II) to evaluate the scales of personality disorders and the IAS as markers of interpersonal space. We found that many personality disorders scales measured the MCMI-II could be identified in the IAS circumplex.
\end{abstract}

Keywords: Personality disorders, Interpersonal behavior, Interpersonal Adjectives Scales (IAS), Interpersonal Circumplex.

\section{INTRODUCCIÓN}

El estudio de la conducta interpersonal comienza a interesar a la investigación sobre los trastornos de la personalidad a partir de la concepción, que aparece en el DSM-III y se mantiene en sus desarrollos posteriores, de los trastornos de la personalidad como exageraciones de rasgos de personalidad normal que se confi-

Recibido 10 enero 2007; aceptado 15 noviembre 2007.

Correspondencia: Universidad de Extremadura, E.U. Enfermería y Terapia Ocupacional, Campus Universitario, Avda. de la Universidad, s/n, 10071 Cáceres (España), Correo-e: efelipe@unex.es guran en un patrón permanente de experiencia interna y comportamiento y se manifiestan, entre otras áreas, en las relaciones interpersonales (e.g. Wiggins y Pincus, 1989).

Este nuevo punto de vista en la conceptualización de los trastornos de la personalidad coincide con los presupuestos de la teoría interpersonal (e.g. Carson, 1969, Leary, 1957) y supone el resurgimiento del interés por la investigación y desarrollo de los modelos dimensionales de evaluación de la personalidad, dentro de los cuales destacamos los modelos circumplex de la conducta interpersonal. Los teóricos interpersonales reconocen que algunas catego- 
rías de trastornos de personalidad reflejan extremos de dimensiones subyacentes de rasgos de personalidad normal y sus teorías proponen representaciones bidimensionales basadas en las coordenadas de dominancia y sostenimiento (Leary y Coffey, 1955).

La primera publicación sobre modelos circumplex en psicología de la personalidad es el trabajo de Freedman, Leary, Ossorio y Coffey de 1951. A partir de este trabajo se desarrollaron una gran cantidad de modelos y teorías que comparten el uso de la estructura circular o circumplex. Uno de los modelos que más investigación está generando, es el circumplex interpersonal (IPC) de J.S. Wiggins. Este modelo cuenta con un instrumento de evaluación, las Escalas de Adjetivos Interpersonales (IAS), construidas en 1979 por Wiggins y revisadas en 1988 por Wiggins, Trapnell y Phillips.

Los modelos circumplex han permitido hacer una relectura de las categorías de los trastornos de la personalidad de los sistemas de clasificación de los trastornos psicopatológicos, abriendo un campo muy interesante de investigación al permitir aclarar su contenido sustantivo interpersonal. En cuanto a su aplicación han demostrado que son modelos útiles no sólo en la evaluación y diagnóstico interpersonal, sino también en la psicoterapia (Benjamín, 1996, Felipe y Ávila, 2005, Wiggins, 2003). En este sentido, según Wiggins (1980), las IAS sirven como marcadores semánticos del espacio interpersonal con objetivos de comparación con otros sistemas de evaluación, y permiten identificar dimensiones de conducta interpersonal en otras escalas e inventarios de evaluación de la personalidad.

La publicación del Eje II del DSM-III provocó esfuerzos importantes para definir los trastornos de la personalidad en estas dos dimensiones interpersonales (e.g. Wiggins, 1982), aunque muchos de estos estudios son más conceptuales que empíricos. Revisaremos algunos de los estudios que han intentado aclarar este contenido sustantivo interpersonal asociado con las categorías de trastornos de la personalidad, aunque aun estamos lejos de llegar a conclusiones definitivas puesto que los trabajos analizados utilizan diferentes grupos poblacionales y diferentes instrumentos de evaluación. En la tabla 1 vemos los resultados obtenidos en algunos de estos trabajos.
Wiggins y Pincus (1989) administraron a 581 estudiantes las escalas de trastornos de la personalidad del MMPI desarrolladas por Morey, Waugh y Blashfield (1985) siguiendo el DSMIII, la lista de trastornos de la personalidad (PACL) de Strack (1987) y el NEO-PI (Costa y McCrae, 1985). Para evaluar el circumplex interpersonal administraron el IASR-B5 (Trapnell y Wiggins, 1990). Encontraron que las categorías de trastornos histriónico, dependiente, esquizoide, evitativo, antisocial y narcisista tenían contenido interpersonal interpretable a partir del circumplex, no así los trastornos de personalidad límite, esquizotípico y compulsivo.

Un trabajo que merece especial atención es el realizado por Wiggins y Broughton (1991), en el que proyectaron en el circumplex interpersonal 24 inventarios y seis escalas de personalidad entre las que estaban escalas del MMPI, el ICL y el Inventario de Depresión de Beck. Utilizaron las escalas IAS originales (Wiggins, 1979) como marcadoras del espacio interpersonal con el objetivo de incrementar su contenido sustantivo. Este trabajo es uno de los escasos estudios cuyos resultados se apoyan en procedimientos de cálculo de la Localización angular, lo que permite su comparación con otros trabajos.

Soldz, Budman, Demby y Merry (1993) replicaron los resultados de Wiggins y Pincus (1989) utilizando la taxonomía del DSM-III-R con un grupo de 102 pacientes que participaban en psicoterapia de grupo. Para evaluar los trastornos de la personalidad administraron el MCMI-II (Millon, 1987) y el Examen de Trastornos de Personalidad (Loranger, 1988). Para evaluar el circumplex interpersonal administraron el Inventario de Problemas Interpersonales (IPI, Horowitz, Rosenberg, Baer, Ureño y Villaseñor, 1988). Encontraron que muchas de las categorías del MCMI-II tenían contenido interpersonal interpretable, excepto las categorías de paranoide, pasivo-agresivo, esquizotípico y masoquista.

Otros autores han publicado trabajos relacionados con el contenido interpersonal de los trastornos de la personalidad, aunque sus resultados hemos de tomarlos con precaución pues no se basan en los análisis trigonométricos propuestos por Wiggins (1996) y que nosotros utilizamos en nuestros análisis. Es el caso de los trabajo de Morey (1985), DeJong, van den 
Tabla 1. Resumen de la localización de las escalas de trastornos de la personalidad en el circumplex interpersonal según diferentes estudios

\begin{tabular}{|c|c|c|c|c|}
\hline $\begin{array}{l}\text { Escala de Trastorno } \\
\text { de la personalidad } \\
\text { (MCMI-II) }\end{array}$ & $\begin{array}{l}\text { Wiggins } \\
\text { (1982) }\end{array}$ & $\begin{array}{c}\text { Wiggins y Pincus } \\
\text { (1989) }\end{array}$ & $\begin{array}{c}\text { Wiggins y } \\
\text { Brougton } \\
(1991) \\
\end{array}$ & $\begin{array}{l}\text { Soldz et al. } \\
\text { (1993) }\end{array}$ \\
\hline Histriónico & LM & PA-NO & PA-NO & $\mathrm{NO}$ \\
\hline Narcisista & $\mathrm{BC}$ & PA-BC & PA & PA \\
\hline Agresivo-sádico** & & & & PA-BC-DE \\
\hline Antisocial & & $\mathrm{DE}$ & $\mathrm{BC}$ & PA-BC-DE \\
\hline Pasivo-agresivo** & $\mathrm{HI}$ & & $\mathrm{DE}$ & \\
\hline Paranoide & $\mathrm{DE}$ & & $\mathrm{DE}$ & \\
\hline Límite & & & & NO \\
\hline \multicolumn{5}{|l|}{ Esquizotípico } \\
\hline Esquizoide & FG & FG & FG & FG \\
\hline Evitativo & & $\mathrm{HI}$ & & FG \\
\hline \multicolumn{5}{|l|}{ Masoquista** } \\
\hline Compulsiva & PA & & & $\mathrm{FG}$ \\
\hline Dependiente & $\mathrm{JK}$ & $\mathrm{HI}$ & & JK \\
\hline
\end{tabular}

Nota $: \mathrm{PA}=$ Seguro-dominante, $\mathrm{BC}=$ Arrogante-calculador, $\mathrm{DE}=$ Frío de ánimo, $\mathrm{FG}=$ Reservado-introvertido, $\mathrm{HI}=\mathrm{Inse}-$ guro-dependiente, $\mathrm{JK}=$ Modesto-ingenuo, $\mathrm{LM}=$ Cálido-agradable y NO= Gregario-extravertido. ** Estas categorías no se mantienen en la DSM-IV.

Brink, Jansen, y Schippers (1989), Sim y Romney (1990) y Pincus y Wiggins (1990).

Con este trabajo pretendemos describir el contenido interpersonal de las categorías de trastornos de la personalidad utilizando las adaptaciones castellanas del MCMI-II e IAS. En primer lugar comprobaremos el cumplimiento del modelo estructural circular por parte de los sujetos clínicos, comparándolo con los datos teóricos y con los datos normativos de la población española. También exploraremos la capacidad del circumplex IAS para representar el contenido interpersonal de las escalas de trastornos de la personalidad del MCMI-II.

\section{MÉTODO}

\section{Participantes}

Los participantes fueron 205 personas que solicitaron consulta por vez primera en un centro de salud mental de la red asistencial pública. De ellos 125 eran mujeres (61\%) y 80 varones (39\%). La media de edad era de 24,2 años (desviación típica: 6,4). Los criterios de exclusión fueron la presencia de sintomatología activa del área psicótica y la incapacidad para cumplimen- tar los autoinformes. En cuanto a los principales problemas indicados por los sujetos el primero más elegido ha sido el relacionado con el estado de ánimo $(37,3 \%)$, seguido de problemas familiares $(18 \%)$, de confianza en sí mismo $(18 \%)$ y problemas de relación interpersonal $(26,7 \%)$.

\section{Instrumentos}

\section{Utilizamos los siguientes cuestionarios:}

Hoja de Datos sociodemográficos y clínicos (Ávila-Espada, 1992). encuesta de datos que se entrega junto con el MCMI-II, o cualquiera de los cuestionarios elaborados por Millon, consta de datos personales y principales problemas que le llevan a consulta.

Escalas de Adjetivos Interpersonales (IAS) (Wiggins, 1996). Autoinforme de 64 adjetivos a los que el sujeto debe responder en que medida le describen como persona en una escala tipo Likert de ocho intervalos. En 1996 se publicó la adaptación al castellano de las IAS realizada por A. Ávila, que mantiene todas las características estructurales y psicométricas del instrumento original (Ávila, Felipe y Gozalo, 1996, Ávila, Llorente, Felipe y Gozalo, 1993, Felipe 
y Ávila, 2002). Existen datos de consistencia interna en población española de las escalas IAS con valores entre 0,719 y 0,904 , para cada una de las ocho escalas. Otros datos de fiabilidad y validez pueden consultarse en el manual del test (Wiggins, 1996). Las IAS están diseñadas para evaluar dos dimensiones fundamentales de las transacciones interpersonales, la dominancia (DOM) y la afiliación o sostenimiento (LOV) $\mathrm{y}$ ofrecen información tanto del estilo interpersonal como de la flexibilidad conductual del sujeto. Además estas escalas fueron construidas para proporcionar marcadores geométricos precisos del contenido interpersonal según un modelo circumplex organizado alrededor de las puntuaciones DOM y LOV como ejes ortogonales. Esto nos permite utilizar las IAS como una estructura taxonómica en la que clasificar otras escalas desarrolladas por otros autores. En la figura 1 podemos ver el modelo geométrico subyacente a las IAS, en la que cada una de las ocho escalas (u octantes) se identifica con una categoría y mediante una designación alfabética (p.ej. seguro-dominante, PA), y cada octante puede ser localizado en el punto medio $\left(90^{\circ}\right)$ o en el sector $\left(67^{\circ}-112^{\circ}\right)$ del circumplex interpersonal. Este perfil interpersonal da información sobre varios aspectos: cómo la persona se describe a sí misma en sus relaciones interpersonales, mediante las puntuaciones que obtiene el sujeto en los ocho octantes, el tipo interpersonal a partir del octante en el que cae

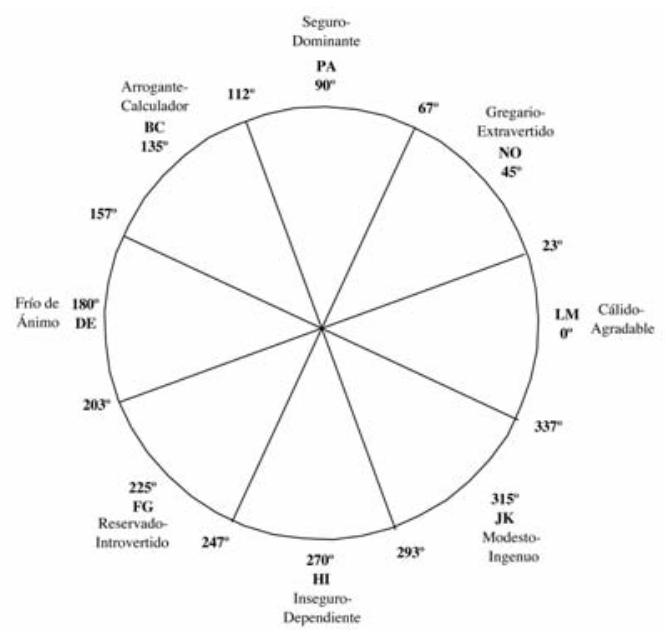

Figura 1. Modelo geométrico de las Escalas de Adjetivos Interpersonales (IAS). (Adaptado de Wiggins y Broughton, 1991). el vector (puntuación de localización angular, LCA, que se calcula a partir de las puntuaciones que obtiene el sujeto en DOM y LOV) y la flexibilidad e intensidad de este tipo interpersonal (puntuación de longitud del vector, LNV).

Inventario Clínico Multiaxial de Millon (MCMI-II) (Millon, 1998). Cuestionario clínico y de personalidad que proporciona información referente a tareas de evaluación y toma de decisiones de tratamiento sobre personas con dificultades emocionales e interpersonales. Formado por 175 ítems, con formato de respuesta verdadero/falso. Está compuesto por 26 escalas, cuatro de validez y fiabilidad y 22 escalas clínicas, en concreto: diez escalas básicas (patrones básicos) de personalidad, tres escalas de trastornos de personalidad patológicos y nueve escalas de síndromes clínicos. Los datos de consistencia interna del cuestionario se sitúan entre 0,90 (escala límite) y 0,63 (escala compulsiva). Otros datos de fiabilidad y validez pueden consultarse en el manual del test (Millon, 1998).

\section{Procedimiento}

Se administraron el cuestionario sociodemográfico, el MCMI-II y las IAS, siempre en este orden, en la primera entrevista, como parte del proceso de evaluación durante una única sesión.

Los análisis estadísticos utilizados son análisis descriptivos y la estimación trigonométrica de los parámetros circumplex, indicados por Wiggins como la metodología apropiada (Wiggins, 1996, Wiggins, Phillips y Trapnell, 1989). Para ello utilizamos el paquete estadístico SPSS-PC.

Analizaremos más detalladamente este procedimiento que nos permite proyectar las escalas del MCMI-II en el circumplex interpersonal de las IAS. Una vez aplicados los instrumentos psicométricos a los participantes, el primer paso es obtener los factores de cada escala del MCMI-II en las coordenadas de la figura 2. El cálculo de los parámetros trigonométricos proporciona puntuaciones exactas de la localización de cada escala en el círculo interpersonal. Para calcular estos parámetros, es necesario conocer el valor de cada escala en las coordenadas del círculo DOM y LOV. Estos valores son la correlación de la puntuación o escala con las puntuaciones DOM y LOV, respectivamente. Las puntuaciones de loca- 
lización angular y la longitud del vector se calculan aplicando las siguientes fórmulas. La localización angular a partir de $q=\tan ^{-1}(y / x)$, y la longitud del vector se calcula a partir de $r=\left(x^{2}+y^{2}\right)^{1 / 2 .}$ Estas dos puntuaciones se utilizan para clasificar las variables o escalas dentro de la taxonomía geométrica. Las escalas de personalidad se distribuyen de forma continua a lo largo del perímetro del circumplex y la localización angular (LCA) nos indica el lugar específico que ocupa dentro del circumplex en relación a las coordenadas de dominancia y sostenimiento. En cuanto a la longitud del vector (LNV) de una escala, formalmente es la correlación múltiple de esa escala con las dos coordenadas ortogonales que definen el espa- cio circumplex, y nos indica el grado en que esa escala puede considerarse interpersonal (Wiggins, 1979), es decir la cantidad de contenido interpersonal que nos ofrece. La puntuación de longitud del vector va de 0 a 1 , en este sentido las escalas con longitud de vector de 0,22 ó superior tendrían, según Wiggins y Pincus (1989), contenido interpersonal interpretable.

\section{RESULTADOS}

En el estudio de la estructura circular de las puntuaciones de los participantes, el primer paso es el cálculo de las intercorrelaciones de los octantes IAS. En la tabla 2 aparecen las

Tabla 2. Análisis correlacional de los octantes IAS

\begin{tabular}{|c|c|c|c|c|c|c|c|c|c|}
\hline \multicolumn{10}{|c|}{ a) Correlaciones entre Escalas IAS en los participantes clínicos. } \\
\hline OCTANTE & PA & $\mathrm{BC}$ & DE & FG & $\mathrm{HI}$ & JK & LM & $\mathrm{N}$ & $\mathrm{O}$ \\
\hline \multicolumn{10}{|l|}{ PA } \\
\hline $\mathrm{BC}$ & 048 & & & & & & & & \\
\hline $\mathrm{DE}$ & 0,29 & 0,48 & & & & & & & \\
\hline FG & $-0,15$ & 0,21 & 0,57 & & & & & & \\
\hline HI & $-0,54$ & $-0,18$ & 0,06 & 0,52 & & & & & \\
\hline JK & $-0,33$ & $-0,37$ & $-0,30$ & $-0,04$ & 0,40 & & & & \\
\hline LM & $-0,10$ & $-0,27$ & $-0,54$ & $-0,41$ & $-0,04$ & 0,56 & & & \\
\hline NO & 0,23 & $-0,08$ & $-0,44$ & $-0,78$ & $-0,46$ & 0,13 & 0,56 & & \\
\hline \multicolumn{10}{|c|}{ b) Correlaciones entre las Escalas IAS, datos normativos españoles (Wiggins, 1996) } \\
\hline OCTANTE & PA & $\mathrm{BC}$ & DE & FG & $\mathrm{HI}$ & JK & LM & $\mathrm{N}$ & $\mathrm{O}$ \\
\hline \multicolumn{10}{|l|}{$\mathrm{PA}$} \\
\hline $\mathrm{BC}$ & 0,44 & & & & & & & & \\
\hline $\mathrm{DE}$ & 0,15 & 0,42 & & & & & & & \\
\hline FG & $-0,10$ & 0,16 & 0,48 & & & & & & \\
\hline HI & $-0,37$ & $-0,08$ & 0,13 & 0,51 & & & & & \\
\hline JK & $-0,26$ & $-0,39$ & $-0,31$ & $-0,10$ & 0,29 & & & & \\
\hline LM & $-0,02$ & $-0,23$ & $-0,55$ & $-0,48$ & $-0,03$ & 0,47 & & & \\
\hline $\mathrm{NO}$ & 0,27 & 0,04 & $-0,37$ & $-0,74$ & $-0,45$ & 0,13 & 0,50 & & \\
\hline
\end{tabular}

\begin{tabular}{|c|c|c|c|c|c|c|c|c|}
\hline Variable & 1 & 2 & 3 & 4 & 5 & 6 & 7 & 8 \\
\hline 1 & 1 & & & & & & & \\
\hline 2 & $\mathrm{R}_{1}$ & 1 & & \multicolumn{3}{|c|}{ donde $\mathrm{R}_{1}>\mathrm{R}_{2}>\mathrm{R}_{3}>\mathrm{R}_{4}$} & & \\
\hline 3 & $\mathrm{R}_{2}$ & $\mathrm{R}_{1}$ & 1 & & & & & \\
\hline 4 & $\mathrm{R}_{3}$ & $\mathrm{R}_{2}$ & $\mathrm{R}_{1}$ & 1 & & & & \\
\hline 5 & $\mathrm{R}_{4}$ & $\mathrm{R}_{3}$ & $\mathrm{R}_{2}$ & $\mathrm{R}_{1}$ & 1 & & & \\
\hline 6 & $\mathrm{R}_{3}$ & $\mathrm{R}_{4}$ & $\mathrm{R}_{3}$ & $\mathrm{R}_{2}$ & $\mathrm{R}_{1}$ & 1 & & \\
\hline 7 & $\mathrm{R}_{2}$ & $\mathrm{R}_{3}$ & $\mathrm{R}_{4}$ & $\mathrm{R}_{3}$ & $\mathrm{R}_{2}$ & $\mathrm{R}_{1}$ & 1 & \\
\hline 8 & $\mathrm{R}_{1}$ & $\mathrm{R}_{2}$ & $\mathrm{R}_{3}$ & $\mathrm{R}_{4}$ & $\mathrm{R}_{3}$ & $\mathrm{R}_{2}$ & $\mathrm{R}_{1}$ & 1 \\
\hline
\end{tabular}

Nota $: \mathrm{PA}=$ Seguro-dominante, $\mathrm{BC}=$ Arrogante-calculador, $\mathrm{DE}=$ Frío de ánimo, $\mathrm{FG}=$ Reservado-introvertido, $\mathrm{HI}=\mathrm{Insegu}-$ ro-dependiente, $\mathrm{JK}=$ Modesto-ingenuo, $\mathrm{LM}=$ Cálido-agradable y $\mathrm{NO}=$ Gregario-extravertido. 
correlaciones de los octantes IAS del nuestro grupo de sujetos participantes junto con los datos normativos obtenidos en la adaptación española de las IAS y la representación de una matriz correlacional circular teórica según Wiggins (1996).

Como podemos observar en la tabla 2 nuestros resultados conforman una representación circular según es postulada por Wiggins (1996), de forma que el rango de las correlaciones va disminuyendo desde puntuaciones positivas elevadas a puntuaciones negativas elevadas a partir de las puntuaciones de la diagonal. La estructura correlacional que obtenemos es similar a la obtenida por Wiggins (1996).

La representación circular de los octantes IAS que obtenemos aplicando los cálculos trigonométricos la podemos ver en la figura 2 , y en la tabla 3 las puntuaciones necesarias para poder situar cada uno de los octantes IAS y el dato más importante, la desviación del valor de la localización angular respecto a la localización teórica propuesta por Wiggins (1996). Los resultados obtenidos con nuestros participantes cumplen el modelo estructural circular postulado por Wiggins (1996). Hay una clara convergencia entre los lugares teóricos y empíricos de las escalas, lo que indica que las IAS utilizadas en población clínica mantienen unas buenas propiedades circumplex, es decir cumplen el modelo estructural postulado.

La única escala que se desvía es la escala seguro-dominante (PA), que lo hace $14^{\circ}$ del lugar teórico en el círculo. Este resultado nos estaría indicando un espacio en la descripción del contenido sustantivo de esa escala.

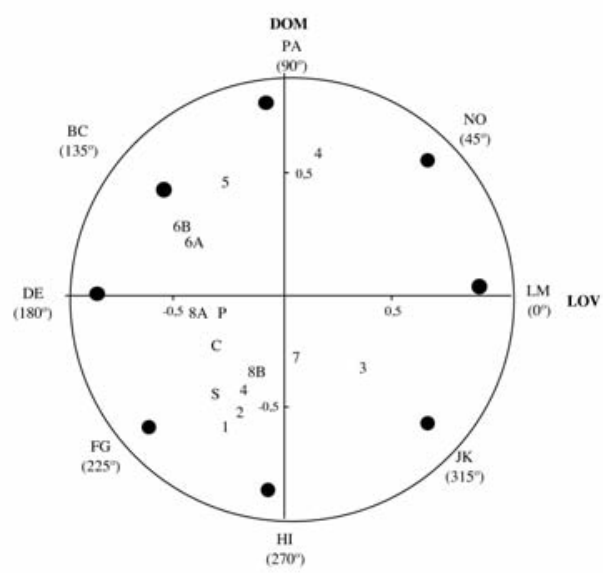

Figura 2. Localización de los octantes IAS y de las escalas de trastornos de la personalidad del MCM-II en el curcumplex interpersonal.

En cuanto al análisis del contenido interpersonal de las escalas de trastornos de la personalidad del MCMI-II, en la tabla 4 podemos ver el cálculo de los valores de cada eje (factores DOM y LOV), la localización angular y el octante IAS al que pertenece cada una de las escalas evaluadas. En la figura 2 vemos proyectadas en el circumplex IAS las escalas cuyo contenido interpersonal es interpretable y en la tabla 1 vemos un resumen de los resultados obtenidos por otros investigadores que nos servirá de apoyo para exponer y analizar nuestros resultados.

En el octante PA localizamos la escala de trastorno de la personalidad histriónico. Este resultado coincide con el obtenido por Wiggins

Tabla 3. Matriz factorial de las Escalas IAS

\begin{tabular}{lccccc}
\hline Escala & DOM & LOV & $\begin{array}{c}\text { Localización } \\
\text { Angular }\end{array}$ & $\begin{array}{c}\text { Localización } \\
\text { teórica }\end{array}$ & Desviación \\
\hline PA Seguro-dominante & 0,74 & $-0,18$ & $104^{\circ}$ & $90^{\circ}$ & $14^{\circ}$ \\
BC Arrogante-calculador & 0,42 & $-0,45$ & $137^{\circ}$ & $135^{\circ}$ & $02^{\circ}$ \\
DE Frío de ánimo & 0,00 & $-0,79$ & $180^{\circ}$ & $180^{\circ}$ & $00^{\circ}$ \\
FG Reservado-introvertido & $-0,59$ & $-0,71$ & $220^{\circ}$ & $225^{\circ}$ & $05^{\circ}$ \\
HI Inseguro-dependiente & $-0,87$ & $-0,10$ & $263^{\circ}$ & $270^{\circ}$ & $07^{\circ}$ \\
JK Modesto-ingenuo & $-0,48$ & 0,54 & $318^{\circ}$ & $315^{\circ}$ & $03^{\circ}$ \\
LM Cálido-agradable & 0,01 & 0,80 & $1^{\circ}$ & $360^{\circ}$ & $01^{\circ}$ \\
NO Gregario-extravertido & 0,60 & 0,71 & $40^{\circ}$ & $45^{\circ}$ & $05^{\circ}$ \\
\hline
\end{tabular}

Nota: DOM: Dominancia, LOV: Sostenimiento. 
Tabla 4. Localización en el circumplex IAS de las escalas del MCMI-II

\begin{tabular}{|c|c|c|c|c|c|}
\hline Escala MCMI-II & DOM & LOV & LNV & LCA & $\begin{array}{l}\text { Octante } \\
\text { IAS }\end{array}$ \\
\hline 1 Esquizoide & $-0,623$ & $-0,281$ & 0,683 & 245,72 & FG-HI \\
\hline 2 Evitativo & $-0,550$ & $-0,234$ & 0,598 & 246,95 & FG-HI \\
\hline 3 Dependiente & $-0,311$ & 0,406 & 0,511 & 307,45 & JK \\
\hline 4 Histriónico & 0,549 & 0,154 & 0,570 & 74,33 & PA \\
\hline 5 Narcisista & 0,436 & $-0,216$ & 0,486 & 116,35 & PA-BC \\
\hline 6A Antisocial & 0,176 & $-0,364$ & 0,404 & 154,19 & BC-DE \\
\hline 6B Agresivo-sádico & 0,193 & $-0,448$ & 0,488 & 156,69 & BC-DE \\
\hline 7 Compulsiva & $-0,252$ & 0,094 & 0,269 & 290,45 & HI-JK \\
\hline 8A Pasivo-agresivo & $-0,024$ & $-0,322$ & 0,323 & 184,26 & DE \\
\hline 8B Masoquista & $-0,364$ & $-0,078$ & 0,372 & 257,9 & $\mathrm{HI}$ \\
\hline S Esquizotípico & $-0,487$ & $-0,269$ & 0,556 & 241,08 & $\mathrm{FG}$ \\
\hline C Límite & $-0,213$ & $-0,266$ & 0,341 & 218,69 & FG \\
\hline P Paranoide & $-0,074$ & $-0,212$ & 0,556 & 199,24 & DE-FG \\
\hline
\end{tabular}

Nota $: \mathrm{DOM}=$ Dominancia, $\mathrm{LOV}=$ Sostenimiento, $\mathrm{LNV}=$ Longitud del vector, $\mathrm{LCA}=$ Localización angular. $\mathrm{PA}=\mathrm{Seguro-}$ dominante, $\mathrm{BC}=$ Arrogante-calculador, $\mathrm{DE}=$ Frío de ánimo, $\mathrm{FG}=$ Reservado-introvertido, $\mathrm{HI}=$ Inseguro-dependiente, $\mathrm{JK}=$ Modesto-ingenuo, $\mathrm{LM}=$ Cálido-agradable y $\mathrm{NO}=$ Gregario-extravertido

y Broughton (1991) quienes localizan la escala histriónica del MMPI también en PA. Los trabajos revisados coinciden en situar esta escala en el cuadrante superior derecho, pero en octantes distintos: NO, LM y PA. El contenido interpersonal asociado con el octante PA hace referencia a personas que se describen a sí mismas como dominantes, asertivas y auto confiadas y tienden a ejercer poder sobre los otros en contextos sociales. Este estilo puede convertirse en autoritarismo y se manifiesta en dificultades interpersonales relacionadas con el control, la manipulación, la agresividad y el intento de cambiar a otros.

La escala de trastorno de la personalidad narcisista la situamos entre los octantes PA (segurodominante) y BC (arrogante-calculador). Nuestros resultados coinciden con los trabajos revisados, por tanto la escala narcisito compartiría características de ambos estilos interpersonales.

Las escalas de trastorno de la personalidad agresivo-sádico y antisocial estarían localizadas entre los octantes BC (arrogante-calculador), y DE (frío de ánimo). Todos los trabajos coinciden en situar estas categorías en torno a los octantes BC y DE. El contenido interpersonal asociado con el octante $\mathrm{BC}$ hace referencia a personas que se describen a sí mismas como egoístas, arrogantes, astutas y explotadoras.
Expresan enfado e irritación en forma de humillación y explotación, con un contenido agresivo importante. Este estilo puede volverse vengativo y se manifiesta a través de problemas relacionados con la desconfianza y la sospecha hacia los otros, así como el desinterés por la felicidad y las necesidades de los demás. Los trastornos agresivo-sádico y antisocial, tendrían componentes del octante DE relacionados con insensibilidad hacia las necesidades de los otros y hostilidad, mientras que el trastorno narcisista tendría aspectos del octante PA relacionados con aspectos de dominancia y control sobre los demás.

La escala de trastorno de la personalidad pasivo-agresivo estaría localizada en el octante DE (frío de ánimo). Hay poco acuerdo respecto al contenido interpersonal que le adjudican diferentes trabajos, que únicamente coinciden en situarle en el lado no afiliativo (izquierdo) del círculo interpersonal.

La escala de trastorno de la personalidad paranoide la localizamos en el octante DE, cercano al FG. En este caso nuestros resultados coinciden con los de otros autores. El contenido interpersonal asociado con el octante DE hace referencia a personas que se describen a sí mismas como no cordiales, ni cálidas, ni amables ni comprensivas. Este estilo puede llevar a 
la persona a ser insensible y se manifiesta a través de problemas que incluyen una incapacidad para expresar afecto y sentir amor hacia otras personas, la dificultad para establecer compromisos duraderos y la inhabilidad para ser generoso y perdonar a los otros. El contenido interpersonal del trastorno paranoide relacionado con el octante FG hace referencia a aspectos de introversión, reserva y timidez, en un esfuerzo por evitar las interacciones sociales y rechazar los acercamientos amistosos de los otros.

Las escalas de trastorno de la personalidad límite y esquizotípico las localizamos en el octante FG (reservado-introvertido). Nuestros resultados son opuestos a los obtenidos por Soldz et al. (1993). El contenido interpersonal asociado con el octante FG hace referencia a personas que se describen a sí mismas como introvertidas, distantes, tímidas y reservadas. Este estilo puede convertirse en rechazo social y se manifiesta a través de una sensación de ansiedad y embarazo en presencia de los otros $\mathrm{y}$ en dificultades para iniciar interacciones sociales, expresar sentimientos y ser sociable.

Las escalas de trastorno de la personalidad esquizoide y evitativo las localizamos en el límite entre los octantes FG y HI. El contenido interpersonal asociado con los octantes FG y HI hace referencia a personas que se describen a sí mismas como introvertidas, distantes, vergonzosas, inseguras y reservadas. Este estilo puede convertirse en rechazo social y se manifiesta a través de una sensación de ansiedad y embarazo en presencia de los otros y en dificultades en dar a conocer nuestras necesidades a los demás, malestar con roles autoritarios e incapacidad para ser asertivo.

La escala de trastorno de la personalidad masoquista la localizamos en el octante HI (inseguro-dependiente). Wiggins (1982) no la incluyó en su trabajo puesto que apareció por primera vez en el DSM-III-R. Soldz et al. (1993) la localizan muy cerca del origen del círculo lo que estaría indicando una ausencia casi total de contenido interpersonal, coincidiendo con Wiggins y Pincus (1989) quienes no la incluyen pues consideran que su proyección en el circumplex interpersonal no es interpretable.

La escala de trastorno de la personalidad compulsiva la localizamos entre los octantes
HI (inseguro-dependiente) y JK (ingenuomodesto). Esta localización es la opuesta a la hipotetizada por Wiggins (1982), en concreto en el octante PA, y tampoco coincide con Soldz et al. (1993) que la sitúan en el octante FG, aunque cerca del origen del circumplex con lo cual su contenido interpersonal no es importante. El contenido interpersonal asociado con el octante HI hace referencia a personas que se describen a sí mismas como tímidas, dóciles, vergonzosas e inseguras o desconfiadas. Carecen de autoestima y autoconfianza y tienen miedo a la evaluación negativa de los otros. Este estilo en su forma problemática muestra incapacidad para ser asertivo, dificultades en dar a conocer nuestras necesidades a los demás y malestar con roles autoritarios. Los sujetos con puntuaciones elevadas en la escalas de trastorno compulsivo de la personalidad se caracterizarían además por ser personas de las que los demás se aprovechan fácilmente e incluso los explotan.

La escala de trastorno de la personalidad dependiente estaría localizada en el octante JK (ingenuo-modesto). Los trabajos revisados coinciden en situar la categoría de trastorno de la personalidad dependiente en el octante JK. Los resultados obtenidos por Wiggins y Pincus (1989) podrían deberse a los contenidos específicos de la escala que mide el MMPI. El contenido interpersonal asociado con este octante hace referencia a personas que se describen a sí mismas como suaves, bondadosas y convencionales. Tienden a ser deferentes, modestas, sinceras y sencillas en las interacciones. Este estilo en su forma problemática se caracteriza por la tendencia a ser explotado y funcionar como alguien que se lo cree todo y del que los demás se aprovechan. No suelen expresar enfado por temor a ofender a los demás.

\section{DISCUSIÓN}

Con este trabajo hemos intentado clarificar la utilidad de las Escalas de Adjetivos Interpersonales (IAS) para la evaluación de la conducta interpersonal en población clínica y analizar el contenido interpersonal de las categorías de trastornos de la personalidad. Para ello hemos 
administrado el MCMI-II, y las Escalas de Adjetivos Interpersonales (IAS) como marcadoras del espacio interpersonal a sujetos con dificultades clínicas y hemos analizado los resultados obtenidos utilizando metodología circumplex. Los resultados obtenidos en nuestro trabajo confirman que las IAS son un instrumento adecuado para la evaluación de la conducta interpersonal con sujetos clínicos, y permiten diferenciar, según el estilo interpersonal característico del sujeto, aspectos clínicos evaluados por el MCMI-II.

La proyección de las escalas de trastornos de la personalidad evaluadas por el MCMI-II en el circumplex interpersonal permite analizar su contenido interpersonal. En este sentido encontramos categorías de trastorno de la personalidad, en algunos casos varias, en todos los octantes del circumplex, excepto en los octantes NO y LM. Considerando los cuadrantes del circumplex, encontramos escalas en todos los cuadrantes, más numerosas en la parte izquierda, no afiliativa, del circumplex y todas las categorías de trastornos de la personalidad están localizadas en lugares del circumplex que tienen una fácil interpretación en términos interpersonales.

El hecho de que varias categorías de trastornos de la personalidad aparezcan en un mismo octante, sugiere que o bien comparten características interpersonales o hay contenidos que el circumplex interpersonal no diferencia o no están representados en el espacio interpersonal. Por ello sería necesario utilizar en estos casos otros conceptos que permitan diferenciarlos, como aspectos cognitivos y afectivos, importantes para lograr una descripción clínica integral de cada categoría de trastorno de la personalidad. En cuanto a los agrupamientos de las categorías de trastornos de la personalidad y comparándolas con los agrupamientos del DSM-IV vemos que, según la localización en el circumplex interpersonal, se confirma el grupo A que incluye las categorías de paranoide, esquizoide y esquizotípico, pero según nuestros resultados podría incluir la categoría de límite y que formarían el cluster excéntrico.

No se confirma el grupo B ya que las categorías de histriónico y límite no aparecen. Es interesante indicar que las categorías de este grupo B antisocial y narcisista, según nuestros resultados, aparecen junto con la agresivo-sádico y pasivo-agresivo, que parece dar importancia a los aspectos agresivos. Estamos de acuerdo con Plutchik y Conte (1994) que habría que renombrar a este agrupamiento como agresivo.

Tampoco se confirma el grupo $\mathrm{C}$, en el que no aparece la categoría de evitativo. Es interesante indicar que las categorías de este grupo $\mathrm{C}$ de dependiente y compulsivo hay aspectos interpersonales comunes y que los diferencian del evitativo. En cuanto a las características interpersonales, este grupo manifiesta un comportamiento que a menudo parece ansioso o temeroso. El que nuestros resultados no confirmen esta agrupación puede tener que ver con que con el circumplex interpersonal no evalúa aspectos relacionados con la ansiedad, que serían probablemente los que nos ayudarían a diferenciarlos.

Si comparamos nuestros resultados con los obtenidos por otros investigadores (ver tabla 1), parece haber acuerdo respecto a la localización de las escalas de trastornos de la personalidad narcisista, agresivo-sádico, antisocial, paranoide, esquizoide, evitativo y dependiente, y poco o ningún acuerdo respecto a las categorías de trastornos pasivo-agresivo, límite, esquizotípico y masoquista. Podemos observar como estas últimas categorías son algunas de las que no se mantienen en la DSM-IV, en concreto pasivoagresivo y masoquista. Si hacemos un análisis de los resultados teniendo en cuenta el cuadrante del circumplex en el que localizamos las categorías, vemos como el cuadrante inferior izquierdo (ver Figura 2) es donde situamos la mayor cantidad de categorías, mientras que en el cuadrante opuesto, el superior derecho, únicamente está el trastorno de la personalidad histriónico. También hemos encontrado lugares del círculo interpersonal descrito por Wiggins, en los que no aparece ninguna categoría, en concreto los octantes LM y NO, que estaría indicando un vacío de la clasificación de los trastornos de la personalidad. La categoría de trastorno de la personalidad depresiva, que el DSM-IV recomienda incluir en investigaciones futuras, no parece que pudiera localizarse en este espacio pues su contenido sustantivo interpersonal no parece ir dirigido hacia el contenido relacionado con estos octantes. 
Las IAS evalúan rasgos interpersonales de la personalidad, es decir, relacionados con los estilos de conducta interpersonal, pero si queremos tener una descripción completa de los trastornos de la personalidad, es necesario evaluar otros contenidos, así como ampliar las categorías de rasgos de la personalidad para poder abarcar todas las posibilidades psicopatológicas que ofrece el circumplex interpersonal. En este sentido sería necesaria una teoría integradora de la personalidad que unifique los resultados obtenidos por diferentes investigadores dándoles una unidad teórica que permita el avance investigador. El modelo de los cinco grandes factores puede proporcionar esa integración. En este sentido Wiggins y Trapnell (1996) consideran que el circumplex interpersonal y el modelo de los cinco grandes son modelos complementarios más que competitivos ya que comparten dos de los factores, en concreto agradabilidad (sostenimiento) y extraversión (dominancia), aunque por otro lado mantienen focos de interés diferente. El modelo circumplex interpersonal permite la investigación más específica del estilo de conducta interpersonal, elemento fundamental de análisis si queremos llegar a comprender los trastornos de la personalidad (Wiggins y Pincus, 1994).

Nuestros resultados apoyan la conclusión de que los factores de dominancia y sostenimiento pueden ayudarnos a diferenciar entre los trastornos de la personalidad, es decir, el estilo de conducta interpersonal puede ayudarnos a diferenciar entre las categorías de trastornos de la personalidad y esta diferenciación sería un apoyo a los modelos dimensionales de evaluación de la personalidad mantenido por numerosos autores (e.g. Widiger, 1991). Finalmente coincidimos con Solz et al. (1993) en que si los trastornos de la personalidad pueden ser considerados como extremos de rasgos normales de la personalidad, porque no podemos evaluarlos en este sentido. El circumplex interpersonal de Wiggins nos aporta un instrumento adecuado de evaluación de uno de los aspectos más relevantes, no sólo para del diagnóstico sino también para la intervención, de los trastornos de la personalidad: el estilo relacional o interpersonal.

\section{REFERENCIAS}

Ávila-Espada, A. (1992). Hoja de datos Sociodemográficos y Clínicos. Monografías de investigación del laboratorio de Psicología Clínica y Psicodiagnóstico. Universidad de Salamanca. Salamanca.

Ávila, A., Felipe, E., y Gozalo, M. (1996). Nuevas aportaciones a la Escala de Adjetivos Interpersonales (SIAS-R): Perfiles y correlatos de los prototipos españoles. En D.E. Gómez y X. L. Saburido (Eds.), Salud y prevención. Nuevas aportaciones desde la evaluación psicológica (pp. 415-422). Universidad de Santiago de Compostela.

Ávila, A., Llorente, A., Felipe, E., y Gozalo, M. (1993). $S I A S-R$. Breve manual para investigaciones previas. Monografías de investigación del Laboratorio de Psicología Clínica y Psicodiagnóstico. Facultad de Psicología. Universidad de Salamanca.

Benjamín, L.S. (1996). Interpersonal diagnosis and treatment of personality disorders $\left(2^{\text {nd }} \mathrm{Ed}\right.$.). New York: Guilford Press.

Carson, R.C. (1969). Interaction Concepts in Personality. Chicago: Aldine.

Costa, P.T., y McCrae, R.R. (1985). The NEO Personality Inventory Manual. Odessa, FL: Psychological Assessment Resources.

DeJong, C.A.J., van den Brink, W., Jansen, J.A.M., y Schippers, G.M. (1989). Interpersonal aspects of DSMIII Axis II: Theoretical hypotheses and empirical findings. Journal of Personality Disorders, 3, 135-146.

Echeburúa. E., y Fernández-Montalvo, J. (2006). Uso y abuso de los autoinformes en la evaluación de los trastornos de la personalidad. Revista de Psicopatología y Psicología Clínica, 11, 1-12.

Feixas, G., Antequera, M., Almuedo, G., y Bach, L. (2006). La estructura cognitiva de los trastornos de la personalidad de evitación y dependiente: Un estudio con la técnica de rejilla. Revista de Psicopatología y Psicología Clínica, 11, 147-154.

Felipe, E., y Ávila, A. (2002). Los perfiles interpersonales: Aspectos clínicos del circumplex interpersonal de Wiggins. Revista de Psicopatología y Psicología Clinica, 7, 19-34.

Felipe, E., y Ávila, A. (2005). Modelos circumplex de la conducta interpersonal: desarrollos recientes y ámbitos de aplicación. Apuntes de Psicología, 23, 183-196.

Freedman, M.B., Leary, T., Ossorio, A.G., y Coffey, H.S. (1951). The interpersonal dimension of personality. Journal of Personality, 2, 171-203.

Horowitz, L.M., Rosenberg, S.E., Baer, B.A., Ureño, G., y Villaseñor, V.S. (1988). The Inventory of Interpersonal Problems: Psychometric properties and clinical applications. Journal of Clinical Psychology, 46, 454459. 
Leary, T. (1957). Interpersonal diagnosis of personality: A functional theory and methodology for personality evaluation. New York: Ronald Press.

Leary, T., y Coffey, H. S. (1954). The prediction of interpersonal behavior in group psychotherapy. Group Psychotherapy, 7, 7-51.

Loranger, A. (1988). Personality Disorder Examination (PDE) Manual. Westchester, NY: Cornell University medical College, Department of Psychiatry.

Millon, T. (1987). Millon Clinical Multiaxial Inventory (MCMI-II). Minneapolis, MN: National Computer Systems.

Millon, T. (1998). Inventario Clínico Multiaxial MCMIII, (adaptación de A. Ávila). Madrid: TEA Ediciones.

Morey, L.C. (1985). An empirical comparison of interpersonal and DSM-III approaches to classification of personality disorders. Psychiatry, 48, 358-364.

Pincus, A.L., y Wiggins, J.S. (1990). Interpersonal problems and conceptions of personality disorders. Journal of Personality Disorders, 4, 340-352.

Plutchik, R., y Conte, H.R. (1994/Junio). The circumplex structure of personality disorders: An empirical study. Poster presentado en el Annual Meeting of the Society for Psychoterapy Research. York, England.

Sim, J.P., y Romney, D.M. (1990). The relationship between a circumplex model of interpersonal behaviours and personality disorders. Journal of Personality Disorders, 4, 329-341.

Soldz, S., Budman, S., Demby, A., y Merry, J. (1993). Representation of personality disorders in circumplex and five-factor space: Explorations with a clinical sample. Psychological Assessment, 5, 41-52.

Strack, S. (1987). Development and validation of an adjective checklist to assess the Millon personality types in a normal population. Journal of Personality Assessment, 51, 572- 587.

Trapnell, P.D., y Wiggins, J.S. (1990). Extension of the Interpersonal Adjective Scales to include the Big Five dimensions of personality. Journal of Personality and Social Psychology, 59, 781-790.

Widiger, T.A. (1991). Personality disorder dimensional models proposed for DSM-IV. Journal of Personality Disorders, 5, 386-398.
Wiggins, J. S. (1979). A psychological taxonomy of trait descriptive terms: The interpersonal domain. Journal of Personality and Social Psychology, 37, 395412.

Wiggins, J. S. (1980). Circumplex models of interpersonal behavior. En L. Wheeler (Ed.), Review of Personality and Social Psychology (pp. 265-294). Beverly Hill: Sage.

Wiggins, J. S. (1982). Circumplex models of interpersonal behaviour in clinical psychology. En C. Kendall y J. N. Butcher (Eds.), Handbook of Research methods in clinical psychology (pp. 183-221). New York: Wiley.

Wiggins, J.S. (1996). Escalas de Adjetivos Interpersonales IAS (adaptación de A. Ávila). Madrid: TEA Ediciones.

Wiggins, J. S. (2003). Paradigms of personality assessment. New York: Guilford Press.

Wiggins, J. S., y Broughton, R. (1991). A geometric taxonomy of personality scales. European Journal of Personality, 5, 343-365.

Wiggins, J. S., Phillips, N., y Trapnell, P.D. (1989). Circular reasoning about interpersonal behaviour: Evidence concerning some untested assumptions underlying diagnostic classification. Journal of Personality and Social Psychology, 56, 296-305.

Wiggins, J. S., y Pincus, A.L. (1989). Conceptions of personality disorders and dimensions of personality. Psychological Assessment, 1, 305-316.

Wiggins, J. S., y Pincus, A.L. (1994). Personality structure and the structure of personality disorders. En P.T. Costa, Jr., y T.A. Widiger (Eds.), Personality disorders and the five factor model of personality (pp. 7393). Washington, DC: American Psychological Association.

Wiggins, J. S., y Trapnell, P.D. (1996). A dyadic-interactional perspective on the five factor model. En J.S. Wiggins (Ed.), The five factor model of personality: Theoretical perspectives (pp. 88-162). New York: Guilford Press.

Wiggins, J. S., Trapnell, P.D., y Phillips, N. (1988). Psychometric and Geometric Characteristics of the Revised Interpersonal Adjective Scales (IAS-R). Multivariate Behavioural Research, 23, 517-530. 